\title{
The ATLAS Trigger System
}

\author{
Cristóbal Padilla on behalf of the ATLAS Collaboration
}

\begin{abstract}
The ATLAS detector at CERN's Large Hadron Collider (LHC) will be exposed to proton-proton collisions at a rate of $40 \mathrm{MHz}$. A three-level trigger system has been designed to select potentially interesting events and reduce the incoming rate to about $200 \mathrm{~Hz}$ of storage rate. The first trigger level is implemented in custom-built electronics; the second and third levels are realized in software. In this paper, the trigger system, its design parameters, and results from the $\mathrm{LHC}$ run in $\mathbf{2 0 0 8}$ are described.
\end{abstract}

Index Terms-Data acquisition, detectors, elementary particles, trigger.

\section{INTRODUCTION}

$\mathbf{T}$ HE ATLAS [1] experiment is one of the two general-purpose experiments installed at the Large Hadron Collider (LHC) at CERN. At the LHC design luminosity $\left(10^{34} \mathrm{~cm}^{-2} \mathrm{~s}^{-1}\right)$, the very short bunch-crossing interval $(25 \mathrm{~ns})$ and approximately 25 overlapping inelastic interactions per bunch crossing make the LHC a very challenging environment that poses severe requirements to the detector design. The trigger system has to reduce the input event rate by more than five orders of magnitude in order to ensure that the data recorded on disk for further analysis mainly contain genuine physics events. The trigger thus must select events whose characteristics indicate that they have been produced in a hard scattering process rather than originating from one of the many soft QCD interactions produced at the LHC. The ATLAS detector accomplishes this task with a three-level trigger system based on regions of interest (RoI). The first-level trigger (L1) is built using custom-made electronics. The other two levels (the High-Level Trigger, HLT) are implemented in software. This paper describes the ATLAS trigger system. Section II details the requirements of the trigger design. Section III describes the global ATLAS Trigger architecture. Sections IV and V give the details of the L1 and the HLT, respectively. Common to the three trigger levels are the configuration database (Section VI) and the monitoring system (Section VII). Section VIII describes the experience of the trigger with the LHC run in 2008. The summary is given in Section IX.

\section{REQUiREMENTS OF THE ATLAS TRIGGER}

The LHC will collide protons at $14 \mathrm{TeV}$ center-of-mass energy. Fig. 1 shows the expected cross sections of several processes of interest at the LHC as a function of the proton-proton

Manuscript received June 05, 2009; revised October 20, 2009. Current version published April 14, 2010.

The author is with the Institut de Física d'Altes Energies (IFAE), Edifici Cn, UAB, E-08193 Bellaterra, Barcelona, Spain, and also with CERN, PH-Department, CH-1211 Geneve 23, Switzerland (e-mail: Cristobal.Padilla@cern.ch).

Color versions of one or more of the figures in this paper are available online at http://ieeexplore.ieee.org.

Digital Object Identifier 10.1109/TNS.2009.2037623

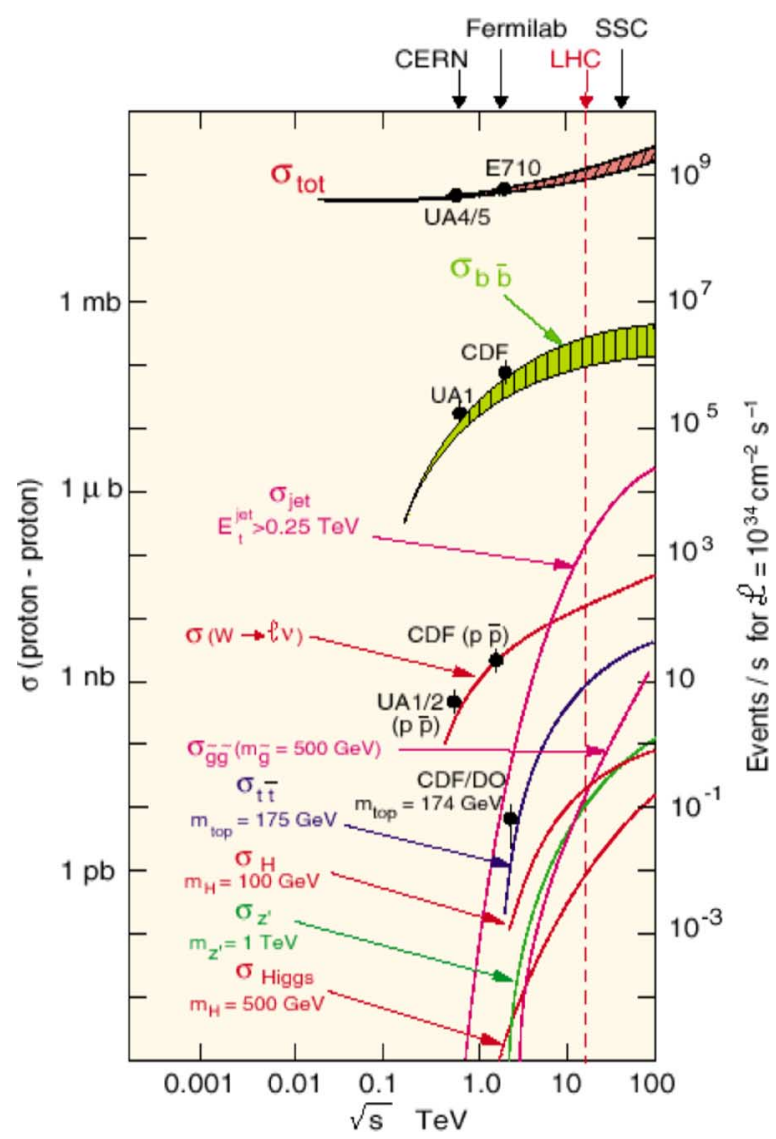

Fig. 1. Cross sections of various processes at the LHC as a function of the center-of-mass energy. The right scale indicates the number of expected events at the maximum LHC design luminosity.

center-of-mass energy. On the right, the expected event rate at the maximum LHC design luminosity is also indicated. Example rates of expected signal events in ATLAS are $0.1 \mathrm{~Hz}$ for a $100 \mathrm{GeV}$ mass Higgs or $0.01 \mathrm{~Hz}$ for a $600 \mathrm{GeV}$ mass Higgs. Supersymmetry events are expected also at around $1 \mathrm{~Hz}$. Interesting b-quark events will be produced at rates of tens of Hertz. At the same time, the background events will be several orders of magnitude higher. Inelastic events will be produced at a rate about $10^{9} \mathrm{~Hz}$, jets with transverse energy above $100 \mathrm{GeV}$ at $\mathrm{kHz}$-rates, and events containing b-quarks at rates of $250 \mathrm{kHz}$. At the same time, the detectors will observe high rates of muons coming from decays of pions, Kaons, and D and B mesons.

The environment at the LHC will also pose several design constraints to the trigger system. It is expected that there will be of the order of 25 overlapping events every $25 \mathrm{~ns}$. At this rate, the number of particles in ATLAS will be, on average, 1400 for every bunch crossing. The trigger has to be designed to detect the few particles that constitute signatures for interesting signal events. 
The trigger system of ATLAS is designed to record the interesting signal events while at the same time rejecting the majority of background; it thereby diminishes the event rate written to tape substantially. To be observed above some background, some signal processes require rejection factors of the order of $10^{13}$.

Other constraints related to the ATLAS detector design determined the architecture of the trigger. With about $10^{8}$ readout channels, the size of an event is of the order of $1.5 \mathrm{MB}$. If all events would be recorded, this event size would result in a storage rate of $50 \mathrm{~TB} / \mathrm{s}$, which is beyond the financial constraints of the experiment. Consequently, the data throughput needs to be reduced to an affordable level, which has been estimated to $200 \mathrm{~Hz}$ on average. At the same time, if we assume that the reconstruction of an event takes $1 \mathrm{~s}, 10^{7} \mathrm{CPU}$ cores would be needed to complete a processing cycle within one year (not counting the needed reprocessing of the same data with improved reconstruction algorithms and calibration and alignment constants). An affordable system requires the reduction of CPU power by, at least, three orders of magnitude.

All LHC experiments fulfill the mentioned requirements using a first-level trigger system based on custom-built electronics that reduces the input rate to the computer farms constituting the higher level trigger. These CPU farms then decrease the event rate to an affordable storage level.

In essence, the trigger system at ATLAS will work at unprecedented regimes in terms of input rate and bandwidth requirements. The L1 input rate to the HLT farms will be one order of magnitude higher than for the Tevatron phase II collider experiments and two orders of magnitude higher than the HERA experiments. At the same time, the fine granularity of the ATLAS detectors increases the event size by, at least, one order of magnitude with respect to these previous experiments.

\section{ATLAS TRIGGER ARCHITECTURE}

ATLAS has chosen a trigger scheme with three distinct levels. Fig. 2 shows the Trigger and Data Acquisition architecture and its internal interactions. Details on the Data Acquisition and controls system can be found in [2]. Here, the aspects related to the trigger system are described in detail.

L1 takes information from the Calorimeter and Muon detectors and has the task to reduce the input bunch crossing rate from $40 \mathrm{MHz}$ to around $75 \mathrm{kHz}$ in a fixed latency of maximum $2.5 \mu \mathrm{s}$, imposed by the length of the on-detector buffers sizes. For those events that are considered interesting, the detector Front-End Electronics sends the data of the corresponding event via the Read-Out Drivers (ROD) to the Read-Out Buffers (ROB) of the Read-Out System (ROS). The bandwidth needed at this step is $120 \mathrm{~GB} / \mathrm{s}$. Events in the ROBs are further processed by the HLT CPU farms in two distinct levels. The Second-Level Trigger (L2) evaluates the event characteristics looking only at a portion of the event data (the L2 takes its decision before the event is fully assembled) and reduces the event rate to around $3 \mathrm{kHz}$ and the bandwidth to the Event Building system to around $5 \mathrm{~GB} / \mathrm{s}$. The $\mathrm{L} 2$ has $40 \mathrm{~ms}$ time to take a decision. The maximum allowed average time per event is basically determined by the size of the L2 farm. The third level of the trigger (so-called Event Filter, EF) performs its selection after the event is fully

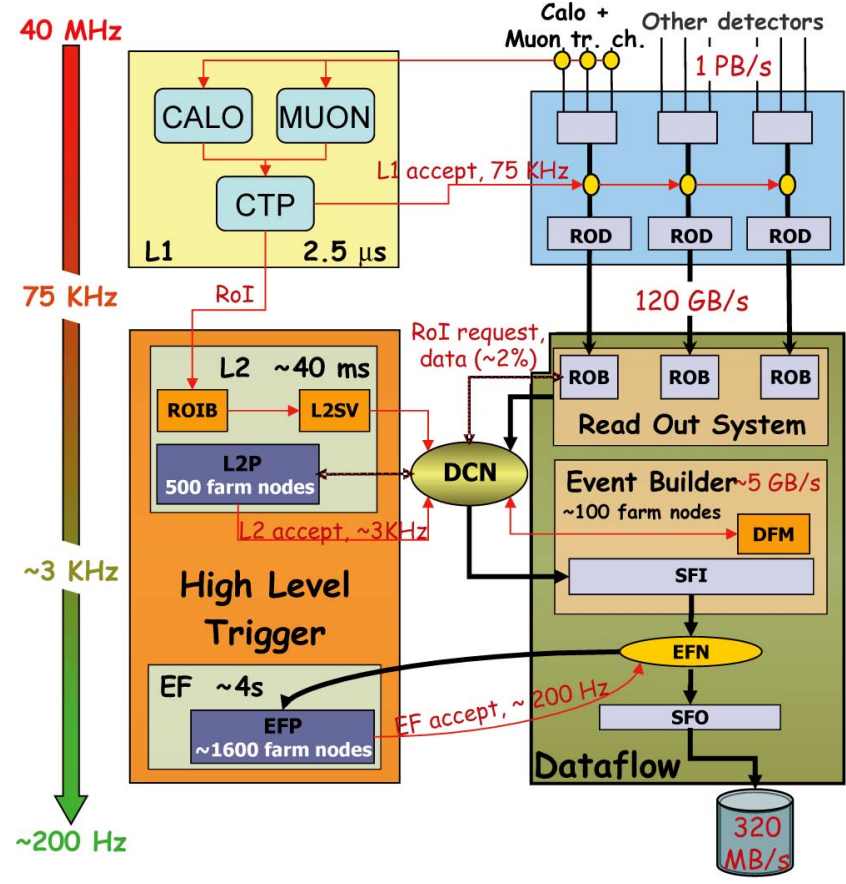

Fig. 2. Schematic view of the ATLAS trigger/DAQ architecture.

assembled in the Event Builder (EB). It reduces the final event rate to $200 \mathrm{~Hz}$ and the mass-storage rate to $320 \mathrm{MB} / \mathrm{s}$. With the currently planned size of the EF farm, the average decision time of the $\mathrm{EF}$ is required to be below $4 \mathrm{~s}$.

Data are transferred to the L2 CPU farms by the so-called RoI mechanism. Fig. 3 gives the details of the data flow of this process. The L1 system identifies geometrical regions of interest on the detector where possible interesting signatures might be located. These include muons, electrons, photons, or jets above a certain transverse energy threshold. The locations in $\eta-\phi$ where these particle candidates are located are collected by the Region of Interest Builder (RoIB) System and transferred to the L2 system. The L1 result is received by the L2 Supervisor (L2SV), which assigns the event for processing to one of the CPU cores in the L2 farm (called a L2 Processing Unit, L2PU). All data of the event resides at this stage in the ROS. The L2PUs execute software algorithms that request event fragments around the RoI specified at L1 in order to confirm the L1 hypothesis. On average, we expect 1.6 RoIs per event (although signal events might have much more RoIs). Consequently, only a few percent of the total event data are transferred to the L2PU in order to take the decision. The $\mathrm{L} 2$ decision is communicated by the L2PU to the L2 Supervisor, which thereafter communicates it to the EB system. If the event is to be accepted, the EB requests all data of the event from the ROS and assembles the complete event in the Sub Farm Input (SFI). After a complete assembling of the event, control is transferred to the Event Filter Data Flow (EFD) system that assigns further processing to an Event Filter Processing Task (EFPT), where the EF selection takes place. Depending on their needs, the EF algorithms unpack the event data that, at this stage, are available through a memory-mapped file stored directly on each EF node such that no network traffic is required to access it. In case of a positive accept decision, the 


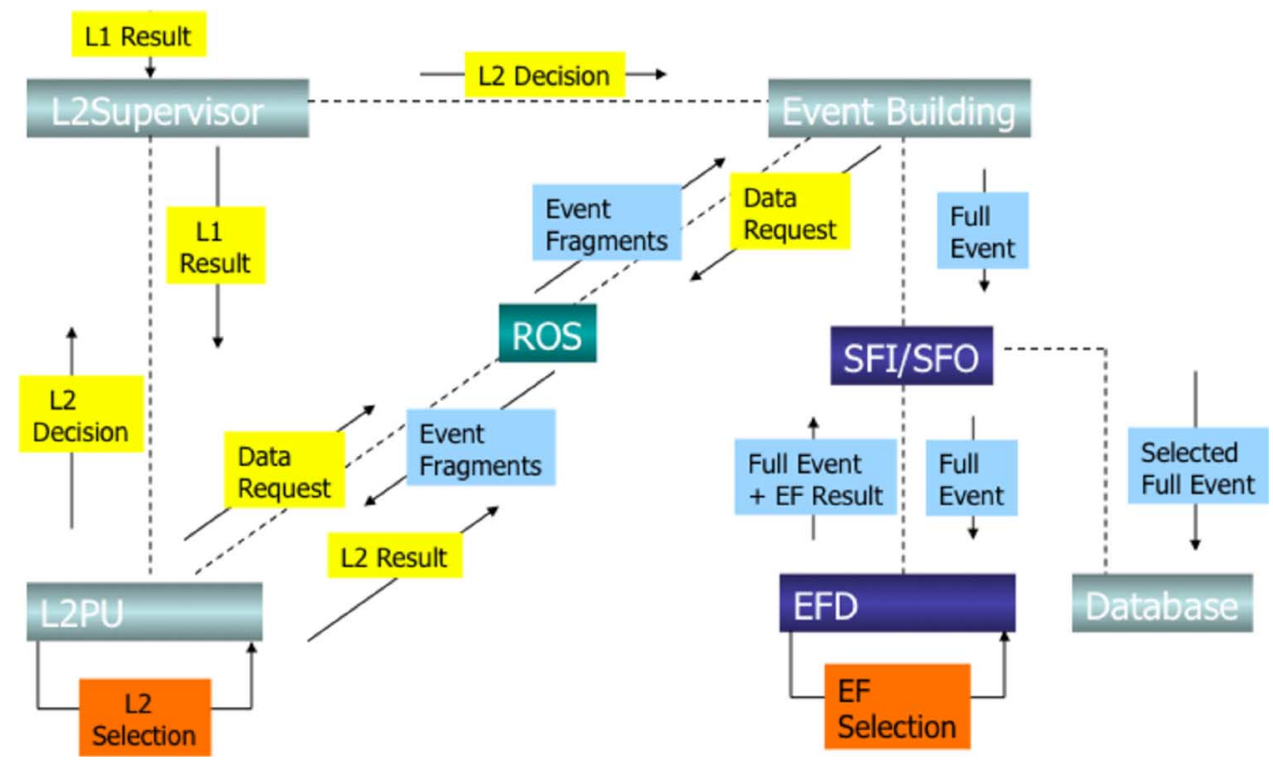

Fig. 3. Data flow of the High-Level Trigger system.

EF result is appended to the event, which is then transferred to the Sub Farm Output (SFO) for final storage.

Most of the element interconnections of the Data Collection Network (DCN) are performed with standard Gigabit Ethernet and switching technology except the S-LINK [3] fiber connections between the RoIB and the L2SV. Each L2SV holds a S-LINK to PCI interface card. Each SFO has a large disk array of $12 \mathrm{~TB}$ and uses three files systems via three RAID disk controllers to maximize the throughput. L2SV, SFI, SFO, L2PU, EFD, and EFPT nodes are multicore PCs with large CPU power and memory size with respect to those used in individual applications.

\section{L1 TRIGGER}

In order to separate the desired rare processes from the predominant QCD jet production and other backgrounds, the L1 system [4] bases its decision on information from the calorimeter and muon systems. The trigger information consists of multiplicities for candidate electrons/photons, taus/hadrons, jets and muons, and of flags indicating that the total scalar transverse energy, the total missing transverse energy, or the total scalar jet transverse energy are above some predefined threshold.

In order to perform its operations fast, the L1 uses reduced granularity data from the muon detectors and calorimeters. For muons, information is retrieved from the Resistive Place Chambers (RPC) and the Thin Gap Chambers (TGC) in the barrel and end-cap region, respectively; calorimetric data from energy depositions of electrons, photon, taus, as well as jets, including missing and total energy sums, are obtained from the different calorimeter parts. The maximal L1 accept rate that the detector readout systems can handle is $75 \mathrm{kHz}$, upgradable to $100 \mathrm{kHz}$.

The 25-ns interval between successive bunch crossings is far too short for processing and selecting events. In fact, given the size of the detectors and with much of the L1 electronics off the detector in a separate cavern, even the transmission delays for the signals are much longer than the bunch-crossing interval. Therefore, the detector data are held in buffers while L1 makes its decision. If the event for a given bunch crossing passes the L1 criteria, a L1 Accept (L1A) signal is sent and the data are transferred to the RODs; otherwise, they are deleted. The maximum latency for the L1 stage depends on the size of the on-detector buffers. In ATLAS, the allowed decision time, or latency, for L1 was chosen to be $2.5 \mu \mathrm{s}$, which is a compromise between long enough processing time to allow effective trigger algorithms to evaluate the event and the cost and complexity of very large data buffers. In order to have a safety margin, the fixed latency of the L1 system was reduced to a maximum of approximately $2 \mu \mathrm{s}$, which also includes the signal transmission delays from and back to the detector front-end electronics.

The overview of ALTAS L1 is shown in Fig. 4. It uses three main components to make its decisions. The L1 Muon Trigger (L1Muon) uses track information from dedicated, fast muon chambers to identify high- $\mathrm{p}_{\mathrm{T}}$ muon candidates. The L1 Calorimeter Trigger (L1Calo) uses calorimeter energy deposits to identify various types of high- $\mathrm{E}_{\mathrm{T}}$ objects as well as energy sums of interest. The Central Trigger Processor (CTP) processes results from both of these systems.

The CTP implements a trigger "menu" based on logical combinations of results from L1Calo and L1Muon. It can also prescale menu items in order to make efficient use of the allowable rate bandwidth as the luminosity and background conditions change.

Events that pass the L1 selection conditions are transferred from the detector-specific front-end electronics to the data acquisition system. In addition, information from L1 itself is read out. In parallel with this, L1 supplies information on the RoIs to the L2 trigger. These RoIs are the geographical coordinates of the detector regions where they were found as well as the criteria (as threshold or isolation conditions) that they satisfied. This information is used by L 2 to seed its selection process. 


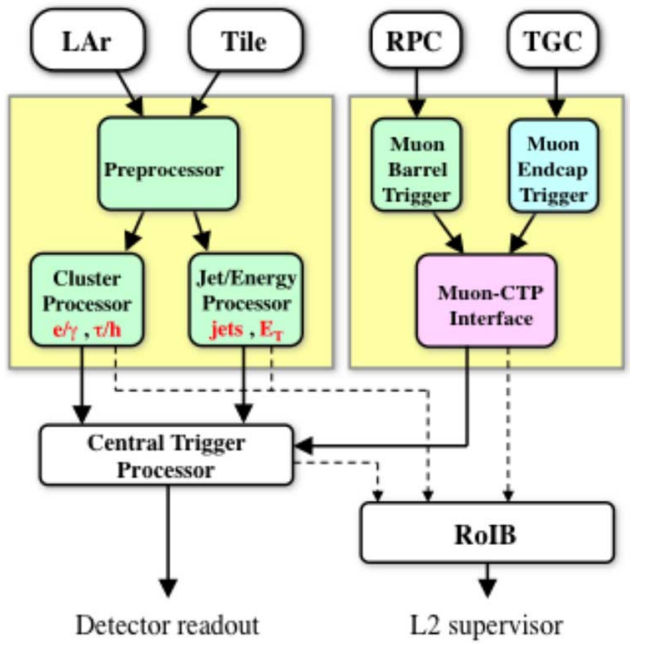

Fig. 4. Overview of the ATLAS Level-1 trigger.

\section{A. L1 Calorimeter System}

The L1Calo [5] is a fixed-latency, pipelined digital system using custom-built electronics. Its input data are summer analog signals from about 7200 so-called trigger towers obtained by grouping cells of the different electromagnetic and hadronic calorimeter parts. Their (reduced) granularity is $0.1 \times 0.1$ in $\Delta \eta \times \Delta \phi(\eta$ is the pseudorapidity, and $\phi$ is the azimuthal angle around the beam axis) for most of the trigger towers. The latency of the calorimeter trigger electronics is less than a microsecond. Including cable transmission delays and the CTP processing time, this results in a total latency of $2.1 \mu$ s for the complete L1Calo chain.

The L1Calo system is located entirely off the detector, in the large separate electronics cavern (USA15); a block diagram of L1Calo's basic architecture is shown in Fig. 5. There are three main subsystems. The PreProcessor first digitizes the analog calorimeter trigger-tower signals, and then uses a digital filtering technique to associate the relatively wide analog pulses with specific LHC bunch-crossings. A lookup table achieves several operations in one step: pedestal subtraction, final transverse energy calibration, noise suppression, and turning off problematic channels. The data are then transmitted in parallel to the two algorithmic processors: the Cluster Processor (CP) and the Jet/Energy-sum processor (JEP). The CP identifies candidate electrons, photons, and taus with high $\mathrm{E}_{\mathrm{T}}$ above programmable thresholds and, if desired, passing predefined isolation requirements. The JEP operates on so-called "jet elements" at the somewhat coarser granularity of $0.2 \times 0.2$ in $\Delta \eta \times \Delta \phi$ to identify jets as well as to produce global sums of total, missing, and jet-sum $\mathrm{E}_{\mathrm{T}}$. Both CP and JEP count multiplicities of the different types of trigger objects and send them, together with bits indicating which global $\mathrm{E}_{\mathrm{T}}$ sum thresholds where exceeded, to the CTP for use in the trigger menu.

For all events that are selected, a programmable selection of data from L1Calo is readout via RODs to the DAQ system. These data include trigger-tower energies and L1Calo results in order to allow calibration, monitoring, and verification of the trigger. Digitized raw data and intermediate results from points

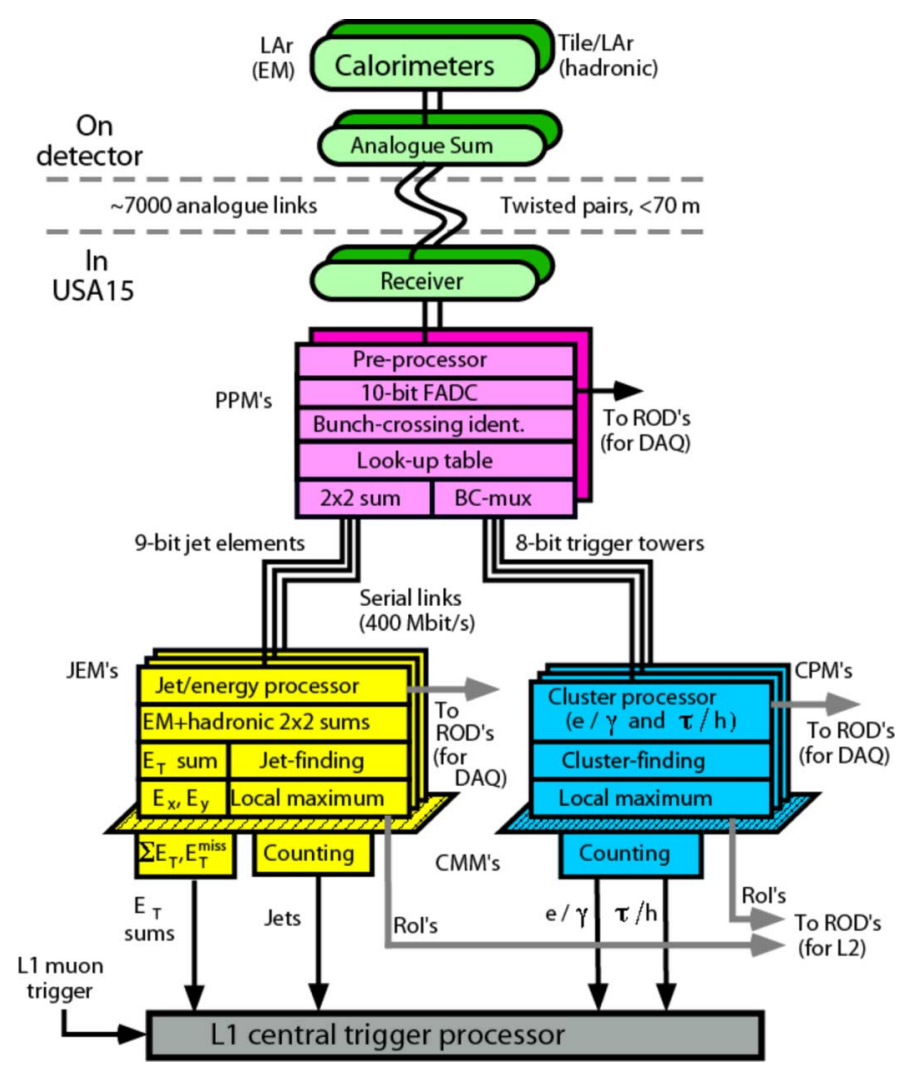

Fig. 5. Architecture of the first-level calorimeter trigger.

along the trigger logic chain will also be read out during commissioning and early running in order to check correct functioning of the trigger and later on whenever they are required to diagnose a problem. The trigger readout data may also produce useful diagnostic information for the LHC machine and ATLAS detectors.

In parallel to the information read out by the DAQ system, RoIs giving details of electron/photon, tau/hadron, and jet cluster candidates are transferred to the RODs and sent to the L2 RoIB; missing, total, and total jet transverse energy values are also sent.

The L1Calo hardware is designed to be relatively compact, with a high density of logic and interconnections. One of the reasons for this is to minimize the latency. In addition, some of the hardware modules are designed to carry out more than one role in the system by using different firmware. This reduces the number of different module types, which in turn leads to a lower hardware cost and simplified maintenance at the price of addition firmware complexity.

The complete L1Calo system has been installed since 2007, all hardware and software has been tested, and calibration procedures with the calorimeters have been exercised. The system has successfully participated in the cosmic and LHC operation data-taking periods in 2008 and 2009.

\section{B. L1 Muon System}

The ATLAS L1 muon [3] trigger selects events with high transverse momentum $\left(\mathrm{p}_{\mathrm{T}}\right)$ muons and assigns the selected candidates to the correct bunch-crossing. Its hardware comprises 


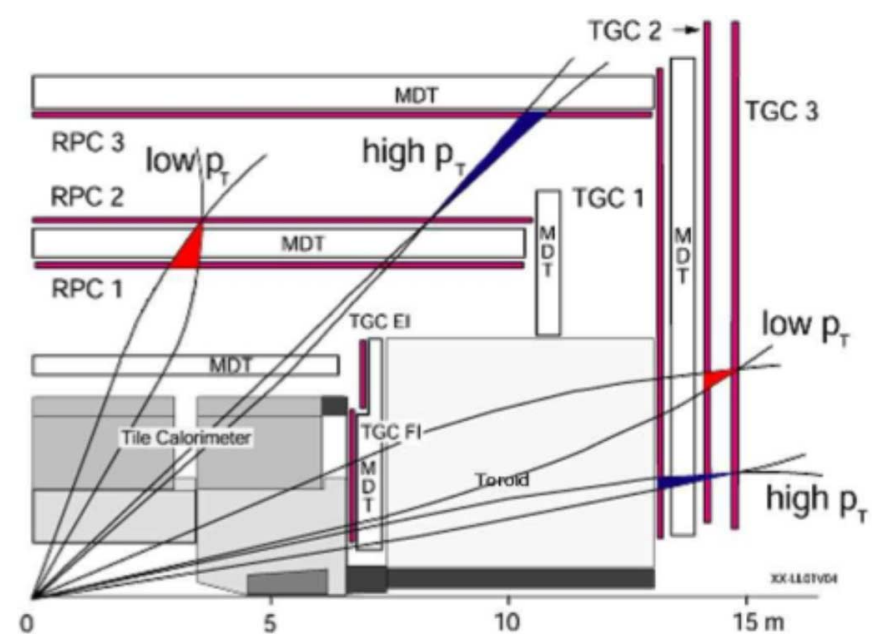

Fig. 6. The L1 selection logic.

electronics for the barrel section instrumented with RPCs and covering the region $|\eta|<1.05$, trigger electronics for the two end-cap regions instrumented with TGCs, and covering $1.05<$ $|\eta|<2.4$ as well as an interface to the CTP (MuCTPI) combining the information from RPCs and TGCs. Using the signals from both RPC and TGC detectors located in the external layers of the ATLAS muon spectrometer, it selects muons with transverse momentum above six programmable thresholds and estimates their approximate position $\eta-\phi$.

The L1Muon selection is based on the definition of allowed geometrical roads so-called Coincidence window $(\mathrm{CW})$. Given a track that hits the middle trigger station (pivot plane), the algorithm searches for time-correlated hits in the confirm plane; there are two confirm planes: one for low and one for high $\mathrm{p}_{\mathrm{T}}$ triggers (see Fig. 6).

A track of infinite momentum originating at the Interaction Point (IP) with direction given by $\eta$ and $\phi$ defines the center of the $\mathrm{CW}$. For each direction, the window size determines a specific $\mathrm{p}_{\mathrm{T}}$ threshold. To calculate the appropriate window size, single muons of the same $\mathrm{p}_{\mathrm{T}}$ as the nominal threshold are simulated. Starting from the center, the window opening in $\eta$ is increased until $90 \%$ of the muons are collected. Tracks of higher transverse momentum will be straighter and consequently will fall into the $\mathrm{CW}$ with higher probability.

The L1Muon electronics is designed to hold three different thresholds for the low and three for the high $\mathrm{p}_{\mathrm{T}}$ configuration. These thresholds are implemented in a FPGA and can be reconfigured to optimize the muon selection at different running conditions.

The on-detector trigger electronics receives as input the hit patterns of the trigger chambers from more than $8 \times 10^{5}$ channels. Coincidences in different trigger stations are identified independently in $\eta$ and $\phi$. The identification is based on geometrical roads whose width is related to programmable $\mathrm{p}_{\mathrm{T}}$ thresholds making use of the deflection of muons in the toroidal magnetic field. In the off-detector part of the muon trigger, the Sector Logic, which is situated in the counting rooms next to the ATLAS cavern, combines the trigger data into muon candidates per trigger sector. For each clock cycle, the muon trigger system sends the obtained sector multiplicities separate for every $\mathrm{p}_{\mathrm{T}}$ threshold to the MuCPTI. The MuCTPI receives information from both RPC and TGC and resolves cases where muon candidates traverse regions with overlapping trigger sectors to avoid double-counting. The resulting final muon multiplicities for the six configurable $\mathrm{p}_{\mathrm{T}}$ thresholds are then sent to the CTP.

The main task of the L1Muon system is the correct identification (within the 25-ns bunch-crossing window) of hits forming a muon candidate. This requires a precise timing of the signals that provide the muon coincidences. The system is fully installed and has been widely used in the recent cosmic and LHC running periods.

\section{Minimum Bias Trigger System}

The Minimum Bias Trigger Scintillators (MBTS) consist of 16 counters per side, of which eight form an inner ring and another eight an outer ring, covering in total a pseudorapidity range of $2.1<|\eta|<3.8$ in the forward direction of each beam. They are mounted at $|\mathrm{z}|=3.6 \mathrm{~m}$ between the Inner Detector and the Liquid-Argon Calorimeter. The signals are read out through the electronics of the hadronic calorimeter, which broaden the fast scintillator signals by roughly a factor of 50 . A second trigger path has been installed to take advantage of the good time resolution of the scintallor. It will allow to distinguish pp-collisions from beam-induced background events by detecting activity of the low transverse momentum particles generated in nondiffractive interactions. A more detailed description of the MBTS and its performance can be found at [6].

\section{Beam-Pickup-Based Timing System}

The beam-pick system (BPTX) [7] consists of electrostatic button pick-up detectors, located $175 \mathrm{~m}$ away along the beam pipe on both sides of ATLAS. They are used both in the trigger system and for the LHC beam monitoring. The BPTX signals are discriminated with a constant-fraction discriminator to provide the a L1 trigger when a bunch passes through ATLAS.

\section{E. L1 CTP System}

The L1 Central Trigger Processor [8] combines information from calorimeter, muon, and other trigger systems and determines the final L1 Accept (L1A) decision on the basis of predefined selection criteria (trigger menus). In addition, the CTP provides trigger summary information to the L2 trigger and the DAQ systems. It also sends L1A rates (accumulated and bunch-by-bunch) for monitoring purposes.

The information received from the L1Calo and L1Muon systems consists of multiplicities for candidate electrons/photon, taus/hadrons, jets and muons, and flags indicating that the total scalar transverse energy, total missing transverse energy, or total jet scalar transverse energy are above some predefined threshold. In addition, it receives inputs from various other sources, including luminosity detectors and beam pickups. The CTP also provides internal trigger signals such as triggers from two random generators or prescaled clocks. Up to 160 input bits can be taken into account by the CTP at any given time.

The CTP generates a L1A signal derived from the trigger inputs according to the $\mathrm{L} 1$ trigger menu. The menu consists of up to 256 trigger items, each of which is a logical combination 
of one or more trigger inputs. Each trigger item can be downscaled, masked, and prioritized individually, where masking allows to select particular bunch configurations (bunch groups), and the priority settings are taken into account in a rather complicated dead-time algorithm that prevents buffer overflow by prohibiting the occurrence of too many consecutive triggers within a certain amount of time (preventive dead time). The L1A is a logical OR of all trigger items after masking and downscaling.

In addition to the final L1 trigger decision, the CTP also provides with each L1A an 8-bit trigger type word, which is sent to all detector front-end electronics via the so-called Trigger Timing Control (TTC) system. It contains bit-encoded information on the trigger type and can be used to steer the event data processing.

For each accepted event, the CTP sends information to the RoIB of the L2 trigger system for guidance of the L2 trigger algorithms. It sends more detailed information to the ROS that can be read out by the DAQ system for events retained after the L2 selection. This information is a superset of the RoI information and can contain data of several bunches before and after the one in which the trigger fired. This feature can be used for debugging and monitoring purposes. The data sent to the RoIB and the ROS provide much more information than the trigger-type word and allows detailed understanding of the reason why an event was selected.

The CTP also provides an absolute GPS-based UTC timestamp that is included in the trigger information sent to the RoIB and the ROS. This allows one to correlate the event to observations from other sources like data taken in other particle or astrophysics experiments.

Several monitoring capabilities have been built into the CTP hardware; it provides snapshots of incoming data, scalers for bunch-by-bunch monitoring of the inputs, and scalers of the trigger inputs and trigger items, before and after prescaling, integrated over all bunches. It also provides the capability of issuing calibration triggers following several mechanisms.

The internal latency of the CTP is $100 \mathrm{~ns}$. The system thus takes four bunch-crossing cycles to issue a L1A decision after receiving the L1 input data.

The CTP hardware consists of the following modules: three input modules (CPT_IN), a bunch-by-bunch monitoring module (CTP_MON), four output modules (CTP_OUT) for sending the L1A decision to the different subdetectors, a machine interface module for clock and timing signals (CTP_MI), and a calibration module for calibration requests from the various subdetectors (CPT_CA).

The complete system is installed and routinely used for datataking since 2007.

\section{High-LeVEL Trigger}

As described in Section III, the HLT is composed of two levels: the Level 2 (L2) and the Event Filter (EF). Fig. 3 describes the aspects of the data movement and application control that are handled by the online data flow and run control software. The selection algorithms run inside the L2 Processing

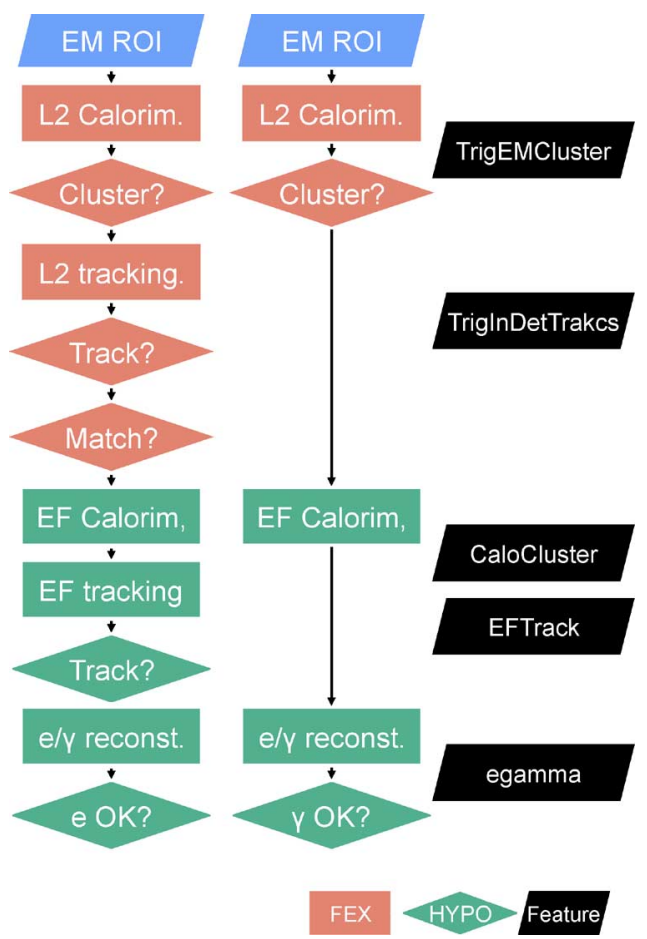

Fig. 7. Example of two trigger chains in the HLT with details of the different algorithm types.

Unit (L2PU) and the EF Processing Task (EFPT). The software framework for both processes is based on the same framework and highly uses software packages developed for offline reconstruction.

The HLT Steering Controller provides for the HLT event selection software the access to the online configuration system and the access to event data, either directly from the ROBs at L2 or from full events in the EF. The L2PUs and EFPTs execute the HLT Steering, which schedules the HLT algorithms for the input seed such that all necessary data for a trigger decision are produced.

Since the EF provides an offline-like, process-based, environment, the HLT event selection software is naturally based on the ATLAS offline reconstruction and analysis environment ATHENA [9]. This allows to efficiently reuse code from a large software basis with many contributors and the implementation of a "physicist-friendly" environment for the trigger algorithm development.

The trigger selection proceeds in steps, called selection sequences, where a feature extraction algorithm reconstructs the physics candidate and a hypothesis algorithm validates it, thereby producing trigger elements. An ordered list of trigger elements constitutes a trigger chain. A complete execution of a trigger chain validates a trigger signature (a required characteristics in an accepted event). Fig. 7 gives examples of two trigger chains validating an electron or a photon. A collection of signatures constitutes the trigger menu. The menu specifies not only the physics selection, i.e. the sequences and the configuration of the related algorithms, but also the processing order of the sequences into the trigger chain. The latter is crucial for optimizing the overall trigger latency and is chosen to prioritize the fastest algorithm because the HLT 

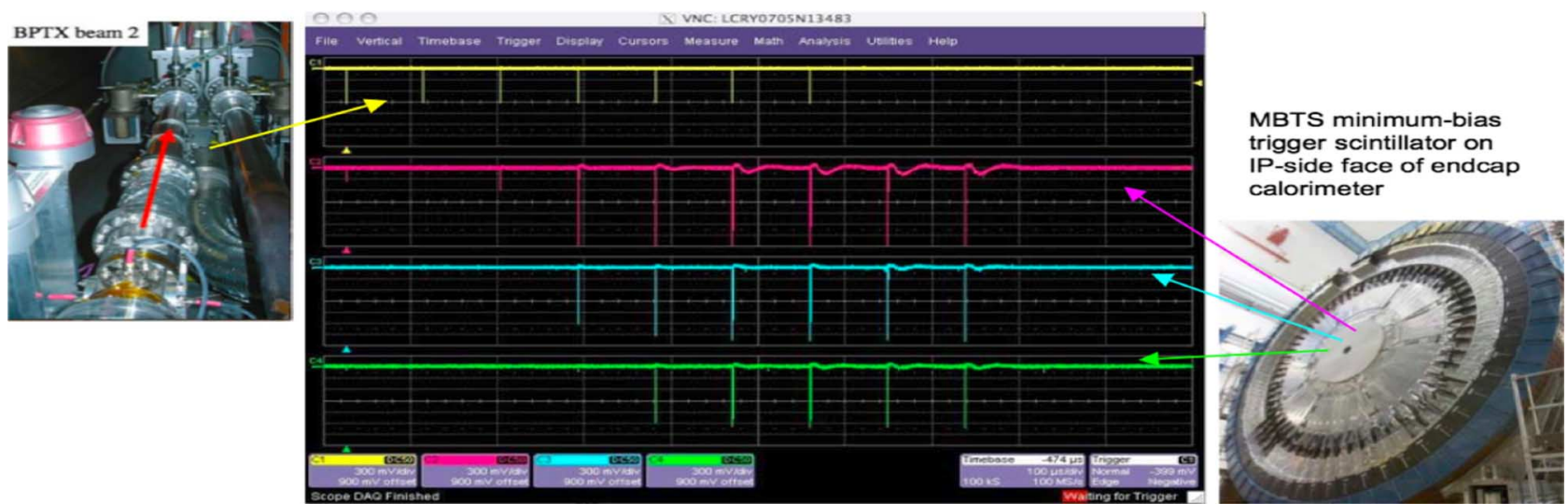

Fig. 8. Activity seen in the beam pickup detectors and the minimum bias scintillators during the first LHC single beam operation.

steering stops further execution of a chain as soon as a selection sequence step fails (early rejection). Further optimization of the trigger latency is performed by the HLT Steering code, which avoids running the same algorithm on a given RoI when an overlap among different trigger chains exists. The system also allows for applying prescales and pass-through factors to the trigger chains in order to keep the event rate under control.

The final HLT system will be composed of about 500 machines for the L2 system and about 1800 for the EF. At present, only 850 nodes (each with dual Harper town quad-core CPUs) are installed in 27 racks. Each node can be configured as L2 or EF node.

\section{Vi. Configuration Database}

The complete ATLAS trigger might contain several hundred selection chains, which requires handling thousands of parameters for configuring the event selection software. Because these parameters, especially those for prescaling and pass-through, are updated constantly to follow the evolution of both the run condition and the detector status, bookkeeping of the full configuration is mandatory. These configuration data are stored in a relational database where the configuration used in a given run is stored in four database keys that specify the L1 and HLT menus and their respective prescale sets. During the run, the menu can be completely changed at a stop/start transition. Prescale sets can be changed at any time during the run without stopping the data-taking process. Finally, the system incorporates many additional tools that allow shifters and experts to interact with the database in a safe manner and to import/export the configuration from/to the offline system, which sets its configuration using XML code.

\section{TRIGGER MONITORING}

Extensive use of histograms is made in the trigger online monitoring system. Their primary use is to assess the quality of the trigger reconstruction and to show the event flow in the system. Those histograms containing information on the reconstruction step are implemented within the corresponding, independently of the trigger chains. Histograms providing information on trigger rates are implemented inside the HLT steering package. All histograms are published via the DAQ control network, and the statistics of every node is collected together by a so-called Gatherer [10] application that presents the combined information to the user. In addition, several applications show the instantaneous trigger rate (and its history) for each L1, L2, and EF trigger item in every step of the configured chains. The algorithm monitoring system has the possibility to perform automatic checks in the histogram contents.

Furthermore, the trigger is also monitored offline by matching the physics objects selected by the L1 system or reconstructed by the HLT with the offline reconstructed results. This process is performed in the CERN Tier0, where the events are reconstructed in less than a day after they have been recorded. More detailed monitoring and debugging is done at the CERN Analysis Facility, which is designed to reprocess $10 \%$ of the recorded event, including rerunning of the HLT selection code. In this processing, the quality of the trigger decision can be assessed by reprocessing the events tagged as minimum-bias physics events or events that have been forced to be accepted at some level of the trigger.

All monitoring tools and procedures have been extensively used during the LHC and cosmic running periods.

\section{EXPERIENCE DuRING THE 2008 LHC RUNnING PERIOD}

During the short running phase in 2008, the LHC machine was operated in several modes yielding different activity in the detectors. In several runs, ATLAS recorded so-called "beam splash" events with huge particle multiplicities. They originate from the collision a single proton beam with tertiary collimators placed $140 \mathrm{~m}$ upstream from the interaction point. When the collimators are closed, a large number of particles flood the detector. The events were triggered mainly using signals from MBTS system positioned on the external surface of the calorimeter cryostat. In addition, the LHC machine provided stable circulation beams (without radio frequency capture) for a maximum period of half an hour. Fig. 8 shows the activity seen in the BPTX and MBTS during these runs. When particles pass through the detectors, they generate signals that were recorded by an oscilloscope. For the first few turns the BPTX signal is clearly seen until the beam gets debunched and defocused; it then gets more evidently detected by the MBTS system.

An important achievement during the first LHC operation was a first synchronization of the trigger signals at the level of one bunch-crossing ( $25 \mathrm{~ns}$ ). Fig. 9 (left) shows the relative trigger 
Relative Trigger Timing, 10 September

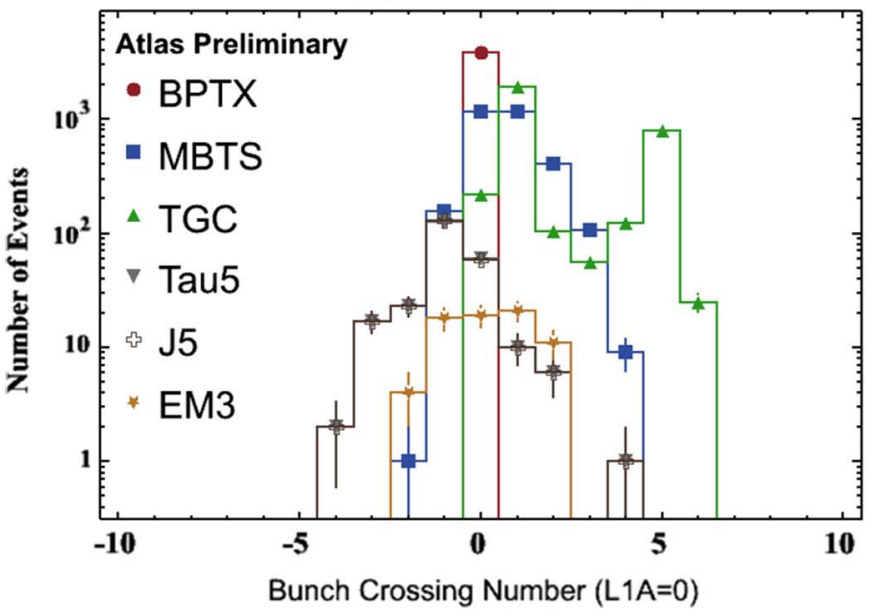

Relative Trigger Timing, 12 September

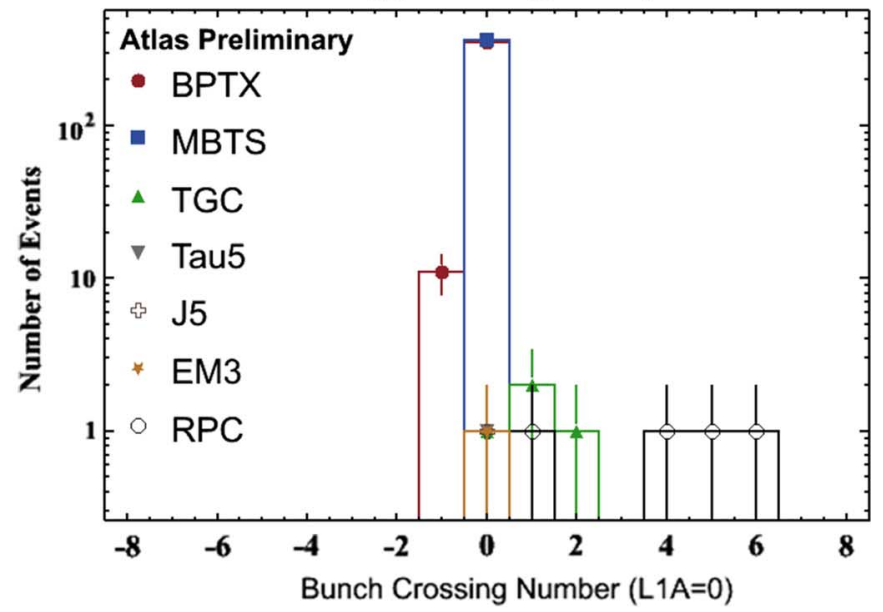

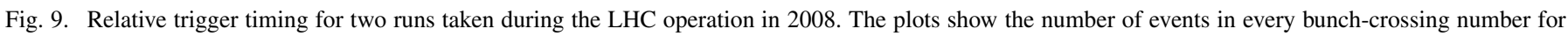
different trigger items.

timing (in number of bunches) for different trigger signals. In particular, the TGC signal shows two peaks separated by four to five bunches, which corresponds to the time of flight of a particle from two ATLAS end-caps and reflects the fact that, during this time, the delays in the various synchronization units were not fully optimized. After two days, the same signals are synchronized, as can be seen in Fig. 9 (right).

It should be pointed out that during this running period, stability and reliability of the trigger system was the main priority. As a consequence, the feature extraction algorithms of the system were not executed in the HLT. Only those algorithms necessary to route the events into the different streams (which were selected according to the $\mathrm{L} 1$ item that triggered the event) were operational.

\section{SUMMARY}

The challenging LHC data-taking environment and practical and budgetary constraints determine the design of the ATLAS trigger architecture. In particular, data reduction factors of more than $10^{5}$ are needed in order to reduce the 40-MHz LHC bunchcrossing rate to an affordable event rate of about $200 \mathrm{~Hz}$ that can be recorded on tape for further analysis.

The ATLAS trigger system is a three-level system. The first level is based on custom electronics and reduces the input LHC rate to $\sim 75 \mathrm{kHz}$ within a fixed maximum latency of $2.5 \mu \mathrm{s}$. The second- and third-level triggers are based on software algorithms (with components based on offline reconstruction packages) using seeds from L1 (RoIs) to guide the HLT levels on the areas of the detector where interesting event signatures can be found. L2 makes its selection before the event building of the complete event has taken place. This results in smaller readout network at the expense of higher complexity for the data control and data traffic software.

The complete trigger system has been extensively tested during the LHC and cosmic ray running periods in 2008, and the equipment is waiting for the first LHC collisions to finalize the commissioning steps and be ready to select interesting physics events that might lead to the discovery of physics beyond the Standard Model within the ATLAS experiment.

\section{REFERENCES}

[1] G. Aad et al., "The ATLAS experiment at the CERN Large Hadron Collider," J. Instrum., vol. 3, p. S08003, 2008.

[2] J. Zhang, "ATLAS data acquisition," in Proc. 16th IEEE NPSS Real Time Conf., Beijing, China, 2009, pp. 240-243.

[3] "CERN S-LINK homepage," [Online]. Available: http://hsi.web. cern.ch/HSI/s-link/

[4] The ATLAS Collaboration, "First-level trigger technical design report," CERN-LHCC-98-014.

[5] R. Achenbach et al., "The ATLAS level-1 calorimeter trigger," J. Instrum., vol. 3, p. P03001, 2008.

[6] R. Kwee et al., "Minimum bias triggers at ATLAS, LHC," in Proc. 34th ICHEP, Philadelphia, PA, Jul.-Aug. 2008, ISA, arXiv:0812.0613.

[7] C. Ohm and T. Pauly, "The ATLAS beam pick-up based timing system," in Proc. 1st Int. Conf. Technol. Instrum. Particle Phys., Tsukuba, Japan, Mar. 2009.

[8] S. Ask et al., "The ATLAS central level-1 trigger logic and TTC system," J. Instrum., vol. 3, p. P08002, 2008.

[9] The ATLAS Collaboration, "ATLAS computing technical design report," CERN-LHCC-2005-22.

[10] P. Renkel, "The gatherer-A mechanism for integration of monitoring data in ATLAS DAQ," in Proc. CHEP, Prague, Czech Republic, 2009. 